\title{
First-principles Study of Phosphorus under High Pressure: Crystal Structure, Superconductivity, and Anharmonicity
}

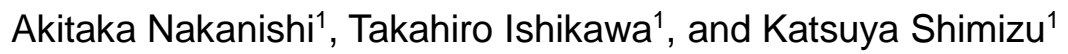 \\ ${ }^{1}$ Department Center for Science and Technology under Extreme Conditions, Graduate \\ School of Engineering Science, Osaka University, Japan
}

E-mail: nakanishi@hpr.stec.es.osaka-u.ac.jp

(Received November 11, 2016)

We have performed first-principles calculations of the structural stability, superconductivity, and anharmonicity of phosphorus under high pressure. The simple hexagonal (sh) structure transforms into an experimentally observed cI16 structure via a body-centered cubic (bcc) structure. In the transition from an sh into bcc structure, the superconducting critical temperature $T_{\mathrm{c}}$ increases from $0.5 \mathrm{~K}$ to $12 \mathrm{~K}$, which is the highest $T_{\mathrm{c}}$ in phosphorus. It decreases to $6 \mathrm{~K}$ in the transition into the cI16 phase. The pressure-dependence of $T_{\mathrm{c}}$ is related to that of the density of states at the Fermi level. In addition, we investigated anharmonicities in phosphorus under high pressure.

\section{Introduction}

Phosphorus takes on the A17 crystal structure (phase P-I) at ambient pressure and transforms to the A7 structure (P-II) at $4.5 \mathrm{GPa}$. Above $10 \mathrm{GPa}$, a simple cubic (sc) structure (P-III) emerges, in which superconductivity exists in the low-temperature range. The superconducting critical temperature $T_{\mathrm{c}}$ reaches a maximum value of $9.5 \mathrm{~K}$ at $32 \mathrm{GPa}$. The sc phase transforms into a simple hexagonal (sh) phase (P-V) via an incommensurately modulated phase (P-IV) [1,2]. A further structural phase transition (P-VI) has been observed at $262 \mathrm{GPa}$ [3]. Though the crystal structure of P-VI had previously been considered to be a body-centered cubic (bcc) lattice, Sugimoto et al. experimentally found, using synchrotron-radiation x-ray powder diffraction [4], that instead it forms a distorted bcc structure (cI16). Conversely, first-principles calculations have predicted that the bcc structure first appears in the P-VI phase and then transforms under further compression into another distorted bcc structure (IM7) with an orthorhombic modulated superlattice [5].

Both the experimental observations and the first-principles calculations ensure the emergence of a distorted bcc phase in phosphorus under high pressure, but consistent results have not yet been obtained. In the present study, we carefully investigated the stability of various crystal phases by comparing the enthalpies among the candidate structures. Based on the resulting sequence of structural phase transitions, we also calculated the superconducting $T_{\text {c. }}$. In addition, we employed the stochastic self-consistent harmonic approximation to investigate the anharmonicity effects for phonons in phosphorus under high pressure.

\section{Computational details}

Structural parameters for the sc lattice, the incommensurately modulated phase IV, and the sh, bcc, cI16, IM7, and hcp lattices at each pressure were obtained by constant-pressure, variable-cell structural optimization based on the Parrinello-Rahman method [6]. For phase IV, we used an approximate structure with a commensurate periodicity of $\gamma=0.250$. The $\boldsymbol{k}$-space integration over the Brillouin zone was performed using a $16 \times 16 \times 16$ Monkhorst-Pack grid [7] for sc, sh, bcc, and hcp, a $16 \times 16 \times 4$ grid for IV, a $24 \times 24 \times 12$ grid for IM7, and an $8 \times 8 \times 8$ grid for cI16. The 
cut-off kinetic energy for wave functions was set at 30 Ry. These $\boldsymbol{k}$-point grids and cut-off energies were chosen to ensure convergence to lower than $0.1 \mathrm{mRy}$ per atom for the total energy. The calculations were performed by the use of the Quantum ESPRESSO (QE) code [8]. We used the exchange-correlation energy functional of Perdew-Burke-Ernzerhof [9] in the generalized gradient approximation and employed the Vanderbilt ultrasoft pseudopotential [10].

We calculated the superconducting $T_{\mathrm{c}}$ using of the Allen-Dynes formula [11] and utilizing the effective screened Coulomb repulsion constant $\mu^{*}=0.1$. We calculated the phonon frequencies by means of density-functional perturbation theory [12,13] on an $8 \times 8 \times 8 \boldsymbol{q}$-point grid for sc, sh, bcc, and hcp, an $8 \times 8 \times 2 \boldsymbol{q}$-point grid for IV, and a $4 \times 4 \times 4 \boldsymbol{q}$-point grid for cI16 using the QE code. The electron-phonon matrix element was calculated using a $32 \times 32 \times 32$ grid for sc, sh, bcc, cI16, and hcp and a $32 \times 32 \times 8$ grid for IV. These grids ensure convergence of the $\boldsymbol{k}$-point sampling with a Gaussian width of $20 \mathrm{mRy}$, which approximates the zero-line-width limits with respect to double-delta functions. To investigate anharmonic phonon effects in the sc structure, we implemented the stochastic self-consistent harmonic approximation (SSCHA) proposed by Errea $e t$ al. [14], within the QE code and used a $2 \times 2 \times 2$ supercell containing 8 atoms .
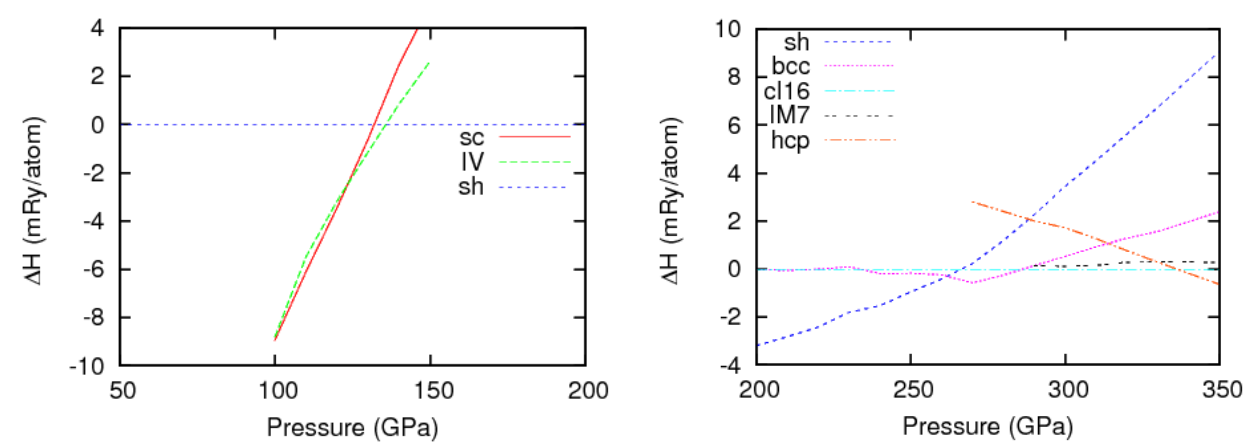

Fig. 1. Enthalpies of sc, IV, sh, bcc, cI16, IM7, and hcp in phosphorus. The relative enthalpies with respect to sh (cI16) are plotted for pressure range of 50-200 GPa (200-350 GPa). The sc phase undergoes structural transition to the IV phase at $120 \mathrm{GPa}$ and to the sh phase at $136 \mathrm{GPa}$. The bcc phase emerges at $262 \mathrm{GPa}$. It transforms to the cI16 phase at $286 \mathrm{GPa}$ and to the hcp phase at $336 \mathrm{GPa}$. The IM7 structure has no stable pressure range up to $350 \mathrm{GPa}$.

\section{Results and Discussion}

\subsection{Sequence of the structural phase transitions}

We first investigated the stability of different crystal structures under high pressure. Figure 1 shows the enthalpies of seven phases (sc, IV, sh, bcc, cI16, IM7, and hcp) with the pressure ranging from 50 to $350 \mathrm{GPa}$. The zero-point energies were not included in the calculations. The calculated stable pressure range of the IV phase, 120-136 GPa, shows a slight discrepancy with respect to that experimentally observed (107-137 GPa) owing to the commensurate approximation used in our calculations. We found that the sh structure transforms into a bcc lattice at $262 \mathrm{GPa}$ and then into the experimentally observed cI16 structure at $286 \mathrm{GPa}$. The earlier-predicted IM7 structure is not stable in any pressure range up to at least $350 \mathrm{GPa}$. Sugimoto et al. reported that the sh phase coexists with the cI16 phase over a wide pressure range from 262 to $322 \mathrm{GPa}$ at room temperature [4]. This suggests that it may be possible to experimentally detect the bcc phase predicted by our calculations by careful investigations in the $262-286 \mathrm{GPa}$ pressure range at low temperatures. The hcp structure emerges as the most stable structure above $336 \mathrm{GPa}$. 


\subsection{Superconducting properties}

Based on the phase diagram obtained above, we investigated the superconducting critical temperature of phosphorus under high pressure. The calculated superconducting transition temperature and the experimentally observed data [15] are shown in Fig. 2. We eliminated the $T_{\mathrm{c}}$ data at pressures where the phonon modes have imaginary frequencies, i.e., the $T_{\mathrm{c}}$ data at $10 \mathrm{GPa}$ in the sc phase and at 270 and $280 \mathrm{GPa}$ in bcc. The calculated $T_{\mathrm{c}}$ values are in good agreement with the experimental values over the range 30-100 GPa, indicating that the calculation results above $100 \mathrm{GPa}$ are also reliable.

Figure 2 shows that $T_{\mathrm{c}}$ decreases from 6 to $3 \mathrm{~K}$ in the transformation from sc to IV, increases to 7 $\mathrm{K}$ in the transformation to sh, and gradually decreases again to almost $0 \mathrm{~K}$ with further pressurization. Through the phase transition from sh to bcc at $260 \mathrm{GPa}, T_{\mathrm{c}}$ increases greatly to $12 \mathrm{~K}$. This value exceeds the experimentally observed value for the sc phase and sets the record for the highest $T_{\mathrm{c}}$ yet obtained for phosphorus. The superconducting $T_{\mathrm{c}}$ decreases to $6 \mathrm{~K}$ in the transition to the cI16 phase and again increases to $8 \mathrm{~K}$ in the transition to the hcp phase.

We also investigated changes in the density of states (DOS) at the Fermi level $N\left(E_{\mathrm{F}}\right)$ during pressurization, which are represented as open triangles in Fig. 2. Here, $N\left(E_{\mathrm{F}}\right)$ was normalized to the unit per atom. The normalized $N\left(E_{\mathrm{F}}\right)$ and $T_{\mathrm{c}}$ show similar pressure dependences above $120 \mathrm{GPa}$, indicating that the change in $N\left(E_{\mathrm{F}}\right)$ is closely related to that of $T_{\mathrm{c}}$. In the modulated structure of the IV phase, the electronic states escape from the Fermi level, and $N\left(E_{\mathrm{F}}\right)$ decreases. The details have been discussed in previous studies $[16,17,18]$. The mechanism of the decrease in $N\left(E_{\mathrm{F}}\right)$ in the IV phase also applies to the cI16 phase. The distorted bcc structure of the cI16 phase creates a pseudogap at the Fermi level. As a result, the cI16 phase has a lower $N\left(E_{\mathrm{F}}\right)$ as compared to either the bcc or hcp phases.

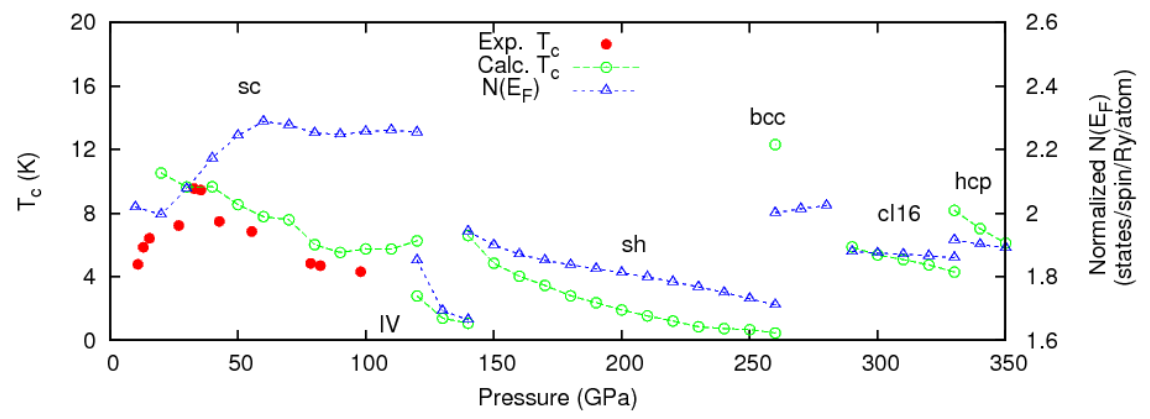

Fig. 2. Pressure dependence of the superconducting critical temperature $T_{\mathrm{c}}$ (open circles) and normalized density of states at the Fermi level $N\left(E_{\mathrm{F}}\right)$ (open triangles) through the sequence of the structural phase transitions: $\mathrm{sc} \rightarrow \mathrm{IV} \rightarrow \mathrm{sh} \rightarrow \mathrm{bcc} \rightarrow \mathrm{cI} 16 \rightarrow$ hcp. Experimentally observed $T_{\mathrm{c}}$ values (solid circles) for the sc phase [15] are also plotted.

Within the harmonic approximation, the calculation results show that the sc phase has imaginary phonon frequencies at $10 \mathrm{GPa}$; this means that the sc structure is mechanically unstable. However, the sc structure is experimentally observed in the pressure range from 10-107 GPa. Anharmonic effects may provide a way to solve this discrepancy between the theoretical and experimental results.

We implemented SSCHA in the QE code and calculated the anharmonic phonon frequencies for the sc structure. The results are shown in Fig. 3. The imaginary phonon frequency at point $\mathrm{R}$, observed within the harmonic approximation, disappears in SSCHA; hence, the sc phase is mechanically stable. 


\section{Conclusion}

In this study, we investigated the stable structures of phosphorus under high pressures, as given in the first-principles calculations. Our results demonstrate that the experimentally observed cI16 structure is stable from 286 to $336 \mathrm{GPa}$ and that the theoretically predicted IM7 structure is unstable up to at least $350 \mathrm{GPa}$.

Based on the phase diagram we obtained, we also calculated the pressure-dependence of the superconducting $T_{\mathrm{c}}$. In the structural transition from sh to bcc at $260 \mathrm{GPa}, T_{\mathrm{c}}$ increases greatly from almost $0 \mathrm{~K}$ to $12 \mathrm{~K}$, which is the highest value of $T_{\mathrm{c}}$ yet obtained for phosphorus. The superconducting critical temperature decreases in the transformation from bcc to cI16 and increases again in the transformation to hcp. The pressure-dependence of $T_{\mathrm{c}}$ was found to be closely related to that of the DOS at the Fermi level $N\left(E_{\mathrm{F}}\right)$.

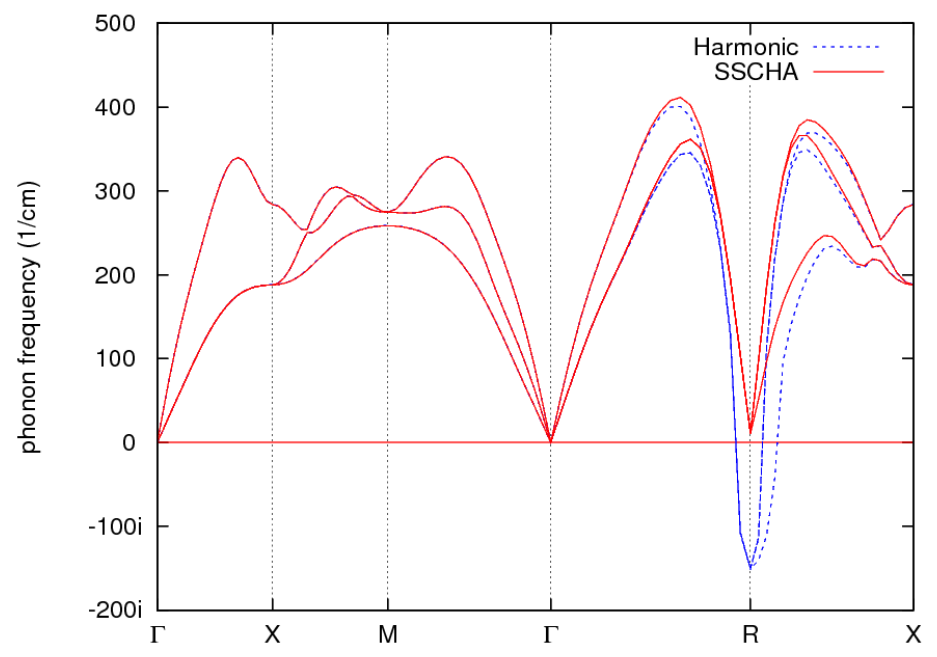

Fig. 3. Phonon-dispersion relations of the sc phase at $10 \mathrm{GPa}$ calculated in both the harmonic approximation and the SSCHA.

\section{Acknowledgment}

This work was supported by JSPS KAKENHI, Grant-in-Aid for Specially Promoted Research (26000006).

\section{References}

[1] T. Ishikawa, H. Nagara, K. Kusakabe, and N. Suzuki, Phys. Rev. Lett. 96, 095502 (2006).

[2] H. Fujihisa, Y. Akahama, H. Kawamura, Y. Ohishi, Y. Gotoh, H. Yamawaki, M. Sakashita, S. Takeya, and K. Honda, Phys. Rev. Lett. 98, 175501 (2007).

[3] Y. Akahama, and H. Kawamura, Phys. Rev. B 61, 3139 (2000).

[4] T. Sugimoto, Y. Akahama, H. Fujihisa, Y. Ozawa, H. Fukui, N. Hirao, and Y. Ohishi, Phys. Rev. B 86, 024109 (2012).

[5] A. S. Mikhaylushkin, S. I. Simak, B. Johansson, and U. Häussermann, Phys. Rev. B 76, 092103 (2007).

[6] M. Parrinello, and A. Rahman, Phys. Rev. Lett. 45, 1196 (1980).

[7] H. J. Monkhorst, and J. D. Pack, Phys. Rev. B 13, 5188 (1976).

[8] P. Giannozzi et al., J. Phys.: Condens. Matter 21, 395502 (2009).

[9] J. P. Perdew, K. Burke, and M. Ernzerhof, Phys. Rev. Lett. 77, 3865 (1996).

[10] D. Vanderbilt, Phys. Rev. B 41, 7892 (1990).

[11] P. B. Allen, and R. C. Dynes, Phys. Rev. B 12, 905 (1975).

[12] S. Y. Savrasov, Phys. Rev. B 54, 16470 (1996).

[13] S. Baroni, S. de Gironcoli, A. D. Corso, and P. Giannozzi, Rev. Mod. Phys. 73, 515 (2001). 
[14] I. Errea, M. Calandra, and F. Mauri, Phys. Rev. B 89, 064302 (2014).

[15] M. Karuzawa, M. Ishizuka, and S. Endo, J. Phys.: Condens. Matter 14, 10759 (2002).

[16] M. Marqués, G. J. Ackland, L. F. Lundegaard, S. Falconi, C. Hejny, M. I. McMahon, J. Contreras-García, and M. Hanfland, Phys. Rev. B 78, 054120 (2008).

[17] T. Ishikawa, H. Nagara, K. Mukose, K. Kusakabe, H. Miyagi, and N. Suzuki, High Pressure Res. 28, 459 (2008).

[18] A. Nakanishi, T. Ishikawa, H. Nagara, K. Shimizu, and H. Katayama-Yoshida, High Pressure Res. 32, 3 (2012). 